\title{
Antivirulence activity of azithromycin in Pseudomonas aeruginosa
}

\author{
Francesco Imperi ${ }^{1+}$, Livia Leoni ${ }^{2+}$ and Paolo Visca ${ }^{2 *+}$ \\ ' Pasteur Institute-Cenci Bolognetti Foundation and Department of Biology and Biotechnology "C. Darwin," "Sapienza" University of Rome, Rome, Italy \\ ${ }^{2}$ Department of Sciences, "Roma Tre" University, Rome, Italy
}

\section{Edited by:}

Rustam Aminov, Technical University

of Denmark, Denmark

\section{Reviewed by:}

Elaine Allan, University College London, UK

Anuradha Ghosh, Kansas State University, USA

${ }^{*}$ Correspondence:

Paolo Visca, Department of Sciences, "Roma Tre" University, Viale G. Marconi 446, 00146 Rome, Italy e-mail: paolo.visca@uniroma3.it

${ }^{\dagger}$ Francesco Imperi, Livia Leoni, and Paolo Visca have contributed equally to this work.
Antibiotics represent our bulwark to combat bacterial infections, but the spread of antibiotic resistance compromises their clinical efficacy. Alternatives to conventional antibiotics are urgently needed in order to complement the existing antibacterial arsenal. The macrolide antibiotic azithromycin (AZM) provides a paradigmatic example of an "unconventional" antibacterial drug. Besides its growth-inhibiting activity, AZM displays potent anti-inflammatory properties, as well as antivirulence activity on some intrinsically resistant bacteria, such as Pseudomonas aeruginosa. In this bacterium, the antivirulence activity of AZM mainly relies on its ability to interact with the ribosome, resulting in direct and/or indirect repression of specific subsets of genes involved in virulence, quorum sensing, biofilm formation, and intrinsic antibiotic resistance. Both clinical experience and clinical trials have shown the efficacy of AZM in the treatment of chronic pulmonary infections caused by $P$. aeruginosa. The aim of this review is to combine results from laboratory studies with evidence from clinical trials in order to unify the information on the in vivo mode of action of AZM in P. aeruginosa infection.

Keywords: antibiotic, cystic fibrosis, inflammation, macrolide, regulation, virulence

\section{INTRODUCTION}

Antibiotics are used as first line drugs for the treatment of bacterial infections, but the widespread resistance to these agents combined with the shortage of novel antimicrobial compounds developed by the pharmaceutical industry results in an urgent need for new strategies to combat bacterial infections (Fernebro, 2011). Virulence factors are essential for bacterial pathogens to cause infection. Hence, suppression of virulence factor production, i.e., antivirulence therapy, has become an attractive anti-infective approach. In-depth understanding of the mechanisms by which pathogens cause disease has been essential for the recognition of suitable targets for antivirulence drugs (Cegelski et al., 2008; Rasko and Sperandio, 2010). Target-based rational design and screening of chemical libraries allowed the identification of a variety of virulence inhibitors (Clatworthy et al., 2007; Law et al., 2013). However, none of the antivirulence compounds developed so far have entered into clinical practice.

The unpredicted antivirulence activity observed a posteriori among macrolide antibiotics prompted revisiting of laboratory and clinical data to assess the potential of these compounds as antivirulence drugs. In this review, the clinical impact of azithromycin (AZM) on patients suffering from Pseudomonas aeruginosa infection are discussed in the light of the biological activities exerted by AZM on both the pathogen and the host.

\section{THE MULTIFARIOUS BIOLOGICAL ACTIVITIES OF MACROLIDES}

Macrolides are polyketide compounds characterized by the presence of a 14- (e.g., erythromycin), 15- (e.g., AZM, Figure 1), or
16- (e.g., josamycin) membered macrocyclic lactone to which one or more amino and/or neutral sugars are attached.

Macrolides have many important biological characteristics including antibacterial, antifungal and immunomodulatory properties. Erythromycin is the progenitor of this class of antibiotics and has served as the scaffold for the generation of newer semisynthetic macrolides (Washington and Wilson, 1985; Pal, 2006). AZM was launched in 1991 and rapidly became one of the most frequently used antimicrobials for outpatients (Hicks et al., 2013). A number of favorable pharmacological properties contributed to the success of AZM as an antibiotic, including acid resistance, a short time to achieve peak concentrations with an up to 800-fold accumulation in phagocytes at the infection site, and long half-life allowing a large single oral dose to maintain bacteriostatic activity in the infected tissue for 4 days (Girard et al., 1987; Foulds et al., 1990; Blumer, 2005).

Macrolide antibiotics inhibit bacterial growth by binding the $23 \mathrm{~S}$ rRNA in the 50S subunit of the bacterial ribosome, thereby preventing the transfer of tRNA from the $\mathrm{A}$ to the $\mathrm{P}$ site of the ribosome. Binding to the A site prevents addition of an incoming amino acid-charged tRNA to the nascent polypeptide chain, ultimately aborting polypeptide growth (Retsema and Fu, 2001; Poehlsgaard and Douthwaite, 2005).

Some macrolides (e.g., rapamycin) lack antibacterial activity but possess potent immunosuppressive or immunomodulatory properties, and are therefore used in the therapy of autoimmune disorders and proliferative diseases. They act through different mechanisms at the level of the immune system, ultimately interfering with lymphocyte activation and cytokine production (McAlister et al., 2002; Ferrer et al., 2011; Salmond and Zamoyska, 2011). 


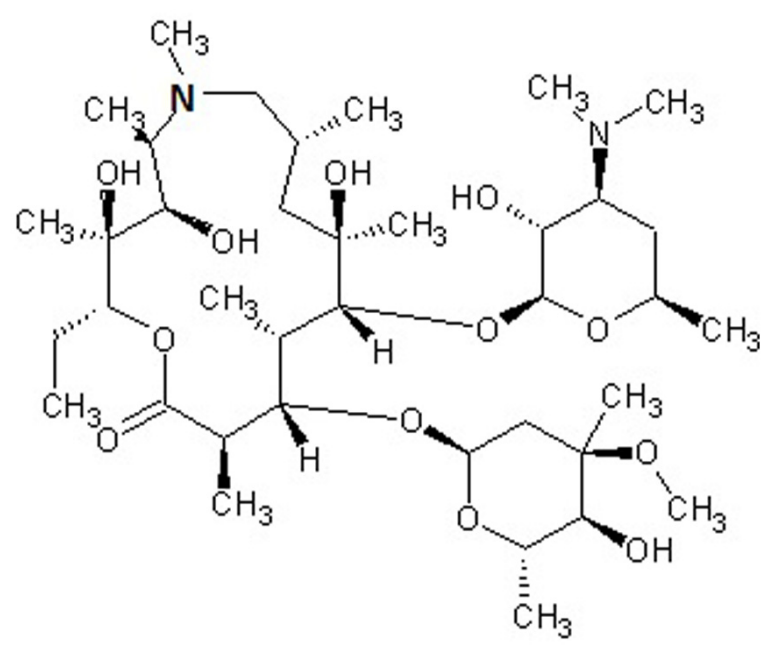

FIGURE 1 | Chemical structure of azithromycin, a drug belonging to the azalide subclass of macrolides. The 15-membered lactone ring is derived from erythromycin, upon incorporation of a methyl-substituted nitrogen atom (bold).

Starting from the late 1960s, evidence has been obtained showing that also macrolide antibiotics have anti-inflammatory and pro-kinetic effects which play a prominent role in some infections (Itkin and Menzel, 1970). These effects have extensively been reviewed in the recent literature (Amsden, 2005; GiamarellosBourboulis, 2008; Kanoh and Rubin, 2010; Steel et al., 2012; Aminov, 2013).

Macrolide antibiotics are typically bacteriostatic at therapeutic concentrations (Retsema et al., 1987). Different from celldisrupting agents (e.g., $\beta$-lactams), they are unlikely to cause bacterial lysis and release of cell-associated pro-inflammatory molecules, thereby avoiding the induction of a detrimental inflammatory response (Spreer etal., 2003; Anderson et al., 2007). Sub-inhibitory concentrations of macrolides cause substantial inhibition of the synthesis of virulence factors in both Grampositive and Gram-negative bacteria (Steel et al., 2012).

Pseudomonas aeruginosa is a paradigmatic example of a microorganism with intrinsic resistance to multiple classes of antibiotics, including macrolides. Nonetheless, a number of clinical studies have demonstrated that patients suffering from both intermittent and chronic $P$. aeruginosa infection, e.g., cystic fibrosis (CF), chronic obstructive pulmonary disease (COPD), and diffuse panbronchiolitis (DPB), benefit from AZM treatment (reviewed by Steel et al., 2012 and Aminov, 2013). Hereafter, the many effects of AZM on P. aeruginosa virulence and their impact on infection are discussed.

\section{EFFECT OF AZITHROMYCIN ON $\boldsymbol{P}$. aeruginosa CELLS}

The pathogenic potential of $P$. aeruginosa relies on the production of cell-surface components with pro-inflammatory and/or adhesion activity, and a huge arsenal of virulence factors (Driscoll et al., 2007). Moreover, its ability to adopt the biofilm lifestyle is critical in chronic infections. In $P$. aeruginosa, the extracellular polysaccharides (EPSs) Psl, Pel and alginate play an important role in maintaining the biofilm structure and in resistance to antibiotics and to the host immune system (Wei and Ma, 2013).

AZM is not approved for the treatment of infections caused by $P$. aeruginosa and there are no published breakpoints for this species. The AZM minimum inhibitory concentrations (MICs) for $P$. aeruginosa range from 8 to $512 \mu \mathrm{g} / \mathrm{ml}$, depending on the strain and the testing procedure (e.g., Kita et al., 1991; Tateda et al., 1996; Nicolau et al., 1999; Morita et al., 2001). Early studies showed that sub-inhibitory AZM concentrations (sub-MIC AZM) suppressed motility and the production of several virulence factors, including proteases, pyocyanin, exotoxin A, phospholipase C (PLC), and EPSs in P. aeruginosa (Kita et al., 1991; Molinari et al., 1992; Molinari et al., 1993; Ichimiya et al., 1996; Nagino and Kobayashi, 1997; Favre-Bonté et al., 2003; Gillis and Iglewski, 2004). Since in $P$. aeruginosa the expression of many virulence factors is activated at the transcriptional level by the 3-oxo-C12-homoserine lactone (3OC12-HSL) and butyryl-homoserine lactone (C4-HSL) quorum sensing (QS) signal molecules, some studies focused on the effect of sub-MIC AZM on these two QS systems.

AZM $(2 \mu \mathrm{g} / \mathrm{ml})$ reduces the production of both 3OC12-HSL and C4-HSL. Transcriptional repression of the corresponding synthase/receptor genes lasI/lasR and $r h l I / r h l R$ contributes to this effect (Tateda et al., 2001). Accordingly, transcriptomic and proteomic analyses confirmed that AZM down-regulates the expression of many QS-dependent genes, as those encoding the pilus, flagellum, and oxidative stress response proteins (Nalca et al., 2006; Skindersoe et al., 2008; Kai et al., 2009). In P. aeruginosa, the AZM-affected transcriptome largely overlaps with the Gac/Rsm regulon, which also includes both las and rhl QS genes (Pérez-Martínez and Haas, 2011). In the Gac/Rsm regulatory pathway, the trans-membrane histidine kinase GacS phosphorylates the response regulator GacA in response to an unknown signal. Phosphorylated GacA activates the transcription of the two small regulatory RNAs (srRNAs) RsmY and RsmZ. At high concentrations, these srRNAs sequester the mRNA-binding protein RsmA, which acts as a translational repressor. RsmA directly or indirectly affects the expression of many virulence genes, including those implicated in QS regulation (Coggan and Wolfgang, 2012; Frangipani et al., 2014). Sub-MIC AZM reduced the expression of several genes in the Gac/Rsm regulon, and inhibited the transcription of the still uncharacterized ORFs PA0588-PA0584, which are required for full expression of $r s m Z$ and $r s m Y$ (Kai et al., 2009; Pérez-Martínez and Haas, 2011). Therefore, AZM-dependent repression of 3OC12-HSL and C4-HSL synthesis could, at least in part, be explained by a cascade mechanism in which AZM represses expression of the PA0588-PA0584 genes which are required for full transcription of $r s m Z$ and $r s m Y$, resulting in down-regulation of QS gene expression. However, the effect of AZM on rsm $Z$ and $r s m Y$ transcription was not completely abrogated in a PA0588-PA0584 deletion mutant, suggesting that AZM affects the Gac/Rsm system and QS also via alternative pathways (Pérez-Martínez and Haas, 2011; Figure 2). Allied to this, AZM also repressed transcription of genes for the synthesis of 3OC12-HSL and C4-HSL precursors (Kai et al., 2009).

Sub-MIC AZM has many pleiotropic effects on $P$. aeruginosa that cannot be explained only by its interference with the Gac/Rsm and QS systems. Examples are (i) the inhibitory effect on alginate 


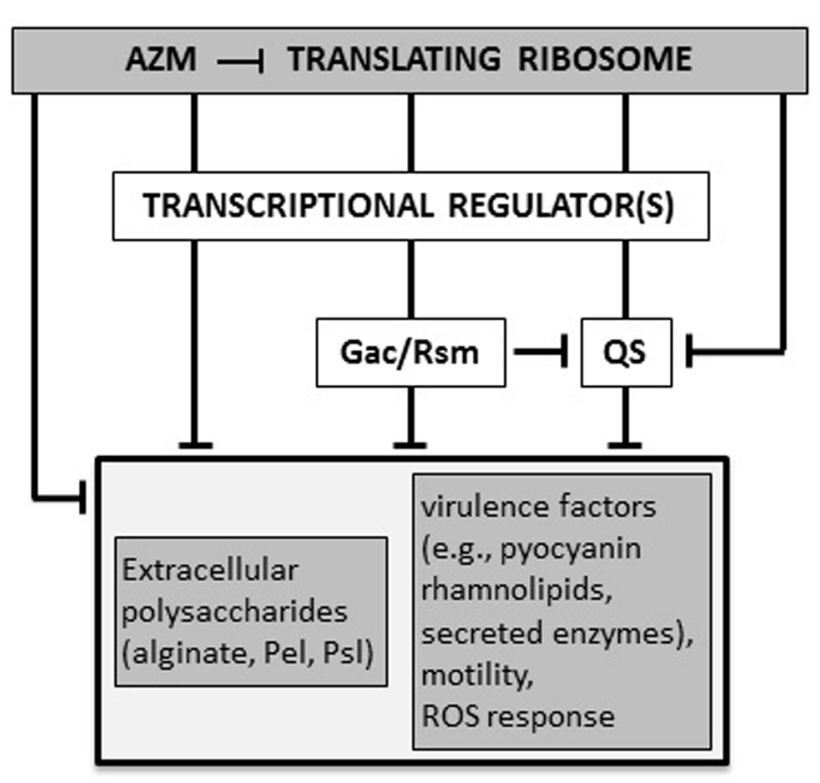

FIGURE 2 | Proposed molecular mechanisms for AZM-mediated inhibition of $\boldsymbol{P}$. aeruginosa virulence. AZM, even at sub-inhibitory concentrations, interacts with the $50 \mathrm{~S}$ ribosomal subunit and selectively affects the expression of a specific subset of genes, such as those for the Gac/Rsm and quorum sensing (QS) systems, and other unidentified transcriptional factors involved in regulation of virulence genes. Some genes (e.g., the QS regulator gene rh/R) may be affected by AZM via independent and overlapping regulatory pathways, both at the transcriptional and post-transcriptional level. The final outcome of this cascade is the suppression of a number of virulence-related phenotypes (see text for details). T-shaped lines represent negative controls.

production (Ichimiya et al., 1996; Favre-Bonté et al., 2003; Lutz et al., 2012), (ii) increased susceptibility to serum bactericidal activity, probably due to alterations of cell-surface structures such as lipopolysaccharides and outer membrane proteins (Tateda et al., 1993, 1994), (iii) increased susceptibility to some antimicrobials, due to down-regulation of the MexAB-OprM efflux pump (Sugimura et al., 2008), and (iv) killing of stationary-phase and biofilm-forming cells (Tateda et al., 1996; Imamura et al., 2005).

Some effects of sub-MIC AZM are dependent on a direct interaction with the ribosome. Indeed, heterologous expression of a Clostridium perfrigens 23S rRNA methylase gene in $P$. aeruginosa increased AZM resistance, counteracted inhibition of virulence factors production, and alleviated killing of stationary-phase cells (Kohler et al., 2007).

Macrolides block elongation of the nascent peptide chain and cause premature dissociation of the tRNA-charged growing polypeptide. Increased release of these abortive peptidyl-tRNAs, a phenomenon known as "drop-off", impairs the normal turnover of tRNAs, affecting the overall protein translation rate (Retsema and Fu, 2001; Gödeke et al., 2013). Interestingly, overexpression in $P$. aeruginosa of the Pth peptidyl-tRNA hydrolase, an enzyme which releases uncharged tRNA from the peptidyl-tRNA, partially restored tRNAs turn-over and reversed some phenotypes caused by sub-MIC AZM, such as the stationary-phase killing and inhibition of pyocyanin and rhamnolipid production (Gödeke et al.,
2013). Therefore, some phenotypes induced by sub-MIC AZM are determined by the increased peptidyl-tRNAs drop-off and defective turn-over of tRNAs. Since production of pyocyanin and rhamnolipids is dependent on the Rhl QS system, Gödeke et al. (2013) by analysing the rhlI and rhlR coding sequences found that the second codon of $r h l R$ (AGG, encoding Arg) is very rarely used in $P$. aeruginosa, suggesting that translation of this gene could be particularly susceptible to defects in tRNAs turn-over. Accordingly, replacement of this codon with the preferentially used codon CGC reverted AZM-mediated inhibition of rhamnolipids and pyocyanin production (Gödeke et al., 2013). This result suggests that sub-MIC AZM may selectively affect the expression of distinct subset of genes, depending on their codon usage. Thus, the pleiotropic effects of sub-MIC AZM on P. aeruginosa are mainly due to AZM interaction with the ribosome and interference with protein synthesis. Differential codon usage in $P$. aeruginosa might explain the selective activity of AZM in translation of specific proteins. Besides RhlR, translation of still unidentified global regulators could also be affected by sub-MIC AZM concentrations, explaining AZM effects on $P$. aeruginosa transcriptome and physiology (Nalca et al., 2006; Skindersoe et al., 2008; Kai et al., 2009).

\section{EFFECT OF AZITHROMYCIN ON $P$. aeruginosa INFECTION}

In Japan, macrolides have been used since the 1980 s to treat DPB, a rare inflammatory lung disease that mainly affects elderly Asian people, in which chronic $P$. aeruginosa lung infection is associated with a poor outcome (Schultz, 2004). In the late 1990s, the similarities between DBP and CF drove Jaffé et al. (1998) to use $\mathrm{AZM}$ as a last resort agent for treatment of a teenager with CF on the waiting list for heart-lung transplantation; AZM treatment almost doubled the patient's pulmonary function, leading to his removal from the list. This promising finding was confirmed by an open-label study on seven CF children infected by $P$. aeruginosa not responding to conventional therapy (Jaffé et al., 1998). Thereafter, several clinical trials have been conducted to validate AZM efficacy in CF. A recent meta-analysis of ten studies, including almost 1,000 patients, showed that AZM therapy is associated with a small but consistent improvement in respiratory function at 6 months, and has a good safety profile (Southern et al., 2012).

Hereafter, results from laboratory studies will be combined with evidence from clinical trials in order to summarize the information on the mode of action of AZM in P. aeruginosa infection. As described above, sub-MIC AZM exerts multiple effects on $P$. aeruginosa, including virulence inhibition, killing of stationary-phase and/or biofilm-forming cells, and synergism with other antimicrobials and with serum complement (Tateda et al., 1996; Imamura et al., 2005; Hoffmann et al., 2007; Lutz et al., 2012). In two chronic lung infection models of CF mice challenged with mucoid (alginate-producing) $P$. aeruginosa isolates, AZM suppressed QS-regulated virulence factors, drastically reduced the bacterial load in the lung, and improved lung pathology (Hoffmann et al., 2007; Tsai et al., 2009). However, in only one study AZM reduced $P$. aeruginosa associated mortality (Tsai et al., 2009). It was also found that AZM attenuated the inflammatory response and promoted macrophage phagocytic activity (Tsai et al., 2009), as previously reported for AZM-treated COPD 
patients (Hodge et al., 2008). The strongly reduced bacterial load in the lungs of AZM-treated mice could be explained by concomitant factors, including killing of biofilm-forming cells, improved phagocytic activity of macrophages, and/or increased susceptibility of QS-attenuated P. aeruginosa to the inflammatory/immune response (Hoffmann etal., 2007; Tsai et al., 2009). However, a reduced bacterial load was not observed for other antivirulence drugs capable of protecting mice from lethal $P$. aeruginosa lung infections (Miyairi et al., 2006; Imperi et al., 2013), suggesting that AZM suppresses the infection by targeting both $P$. aeruginosa and the immune system. Accordingly, relevant antiinflammatory effects of AZM were also observed in CF mice that were not infected with $P$. aeruginosa, where AZM treatment resulted in attenuated cellular infiltration and reduced cytokine release (Legssyer et al., 2006). Therefore, the anti-inflammatory properties of AZM in the lung are also independent of its antiPseudomonas activity. It should be noted that previous work using non-CF murine models of lethal sepsis or pneumonia caused by non-mucoid and mucoid $P$. aeruginosa isolates, respectively, failed to show protective effects of AZM alone, although AZM acted synergistically with ceftazidime in both infection models (Nicolau et al., 1997, 1999). This suggests that experimental conditions have a considerable impact on the outcome of AZM treatment in animal infection models and/or that special features of CF lungs could contribute to improved AZM activity on $P$. aeruginosa.

The therapeutic efficacy of AZM in CF has been proven in many clinical trials. Beneficial effects were observed in CF patients chronically-infected with $P$. aeruginosa and, to a lesser extent, in uninfected CF patients (reviewed in Southern et al., 2012). The latter observation is consistent with the finding that AZM significantly reduced various serum inflammatory markers in CF patients not infected with $P$. aeruginosa (Ratjen etal., 2012), confirming again that AZM has anti-inflammatory effects independent of its antivirulence activity. However, some pulmonary function parameters, including forced expiratory volume in 1s (FEV1), were slightly less improved in patients without chronic $P$. aeruginosa infection compared to chronically-infected patients (Southern et al., 2012). Whether different outcomes are related primarily to the anti-Pseudomonas activity of AZM or to the early stage of lung disease in young patients uninfected with $P$. aeruginosa (Clement et al., 2006; Saiman et al., 2010) cannot be established at present.

Regarding the antivirulence activity of AZM in humans, a retrospective study observed a correlation between the inhibitory effect of AZM on PLC production by $P$. aeruginosa strains isolated from $\mathrm{CF}$ patients and the observed FEV1 improvement after AZM therapy (Nguyen et al., 2007), suggesting that in vivo PLC production is a main target of AZM. This finding fits well with the relevant role of $P$. aeruginosa PLC in the impairment of lung function in a mouse infection model (Wargo etal., 2011). A more recent study in intubated patients colonized with $P$. aeruginosa attempted to directly correlate the clinical effect of AZM on the patient with its antivirulence activity (van Delden et al., 2012). Although no relevant differences were observed between AZM-treated and untreated patients with regard to the occurrence of ventilator-associated pneumonia (VAP), a lower incidence of VAP was reported in a small sub-group $(n=5)$ of AZM-treated patients infected by P. aeruginosa strains producing high levels of rhamnolipids compared with the corresponding untreated group. Although preliminary, this observation suggests that AZM could be more effective in individuals infected by these highly virulent strains (van Delden et al., 2012).

Only few studies measured AZM levels during administration to CF patients. Data so far available suggest a wide range of AZM concentrations in sputa $(0.6-79.3 \mu \mathrm{g} / \mathrm{ml})$, depending on the individual patient and the dosing regimen (Baumann et al., 2004; Wilms et al., 2006). As discussed above, the growth inhibitory activity of AZM is strongly influenced by culture conditions. Since AZM MICs for $P$. aeruginosa are low $(2-16 \mu \mathrm{g} / \mathrm{ml})$ when determined in eukaryotic cell media or in mouse bronchoalveolar lavage fluid (Buyck et al., 2012), it may be possible that AZM also exerts some inhibition of $P$. aeruginosa growth in CF lungs. However, the insignificant differences in the frequency and concentration of $P$. aeruginosa in sputa from AZM-treated and untreated patients (Equi et al., 2002; Saiman et al., 2003; Clement et al., 2006) argue against this possibility.

\section{CONCLUSION}

From a microbiological perspective, the therapeutic efficacy of an antimicrobial compound results mainly from its ability to impair bacterial growth, and this assumption has driven antibiotic research until now. AZM provides a clear example of how the therapeutic efficacy of an antimicrobial cannot exclusively be attributed to growth impairment. Anti-inflammatory and antivirulence properties likely predominate in the treatment of infections involving AZM-resistant pathogens, as in the case of $P$. aeruginosa pulmonary infections. Although there is strong evidence of antivirulence activity in vitro, it is not possible to assess the contribution of this activity to the efficacy of AZM in vivo because of concomitant anti-inflammatory activity and bactericidal effects under certain conditions. According to the current model, both antibacterial and antivirulence activities are based on the interaction of AZM with the ribosome, so that tightly interwoven effects on bacterial viability and production of virulence factors are hardly distinguishable in vivo.

Beneficial effects have so far been documented in CF patients treated with AZM for up to 6 months, while reduced efficacy was associated with longer treatment duration (Southern et al., 2012; Fleet et al., 2013). Loss of efficacy could be explained by the emergence of $P$. aeruginosa subpopulations that become insensitive to the antivirulence activity. This hypothesis could be verified by testing the virulence properties and the response to AZM in serial P. aeruginosa isolates collected during long-term AZM therapy. Under this condition, the emergence of macrolide resistance among both commensal bacteria and co-infecting pathogens is a matter of concern and deserves further study (Aminov, 2013).

In conclusion, AZM offers a unique model to reconsider the central dogma of antibiotic activity, but further research is needed to gain more insight into the effects of AZM on both the pathogen and the host. 


\section{ACKNOWLEDGMENTS}

We thank Prof. H. Seifert for critical reading of the manuscript. Francesco Imperi is supported by the Italian Cystic Fibrosis Research Foundation (grant FFC\#10/2013) and the Pasteur Institute-Cenci Bolognetti Foundation. Livia Leoni and Paolo Visca are supported by grants from the Italian Cystic Fibrosis Research Foundation (grant FFC\#10/2013) and the Italian Ministry of University and Research - PRIN 2012 (prot. 2012WJSX8K), respectively.

\section{REFERENCES}

Aminov, R. I. (2013). Biotic acts of antibiotics. Front. Microbiol. 4:241. doi: 10.3389/ fmicb. 2013.00241

Amsden, G. W. (2005). Anti-inflammatory effects of macrolides - an underappreciated benefit in the treatment of community-acquired respiratory tract infections and chronic inflammatory pulmonary conditions? J. Antimicrob. Chemother. 55, 10-21. doi: 10.1093/jac/dkh519

Anderson, R., Steel, H. C., Cockeran, R., von Gottberg, A., de Gouveia, L., Klugman, K. P., et al. (2007). Comparison of the effects of macrolides, amoxicillin, ceftriaxone, doxycycline, tobramycin and fluoroquinolones, on the production of pneumolysin by Streptococcus pneumoniae in vitro. J. Antimicrob. Chemother. 60 , 1155-1158. doi: 10.1093/jac/dkm338

Baumann, U., King, M., App, E. M., Tai, S., König, A., Fischer, J. J., et al. (2004). Long term azithromycin therapy in cystic fibrosis patients: a study on drug levels and sputum properties. Can. Respir. J. 11, 151-155.

Blumer, J. L. (2005). Evolution of a new drug formulation: the rationale for highdose, short-course therapy with azithromycin. Int. J. Antimicrob. Agents 3, S143S147. doi: 10.1016/S0924-8579(05)80320-6

Buyck, J. M., Plésiat, P., Traore, H., Vanderbist, F., Tulkens, P. M., and Van Bambeke, F. (2012). Increased susceptibility of Pseudomonas aeruginosa to macrolides and ketolides in eukaryotic cell culture media and biological fluids due to decreased expression of oprM and increased outer-membrane permeability. Clin. Infect. Dis. 55, 534-542. doi: 10.1093/cid/cis473

Cegelski, L., Marshall, G. R., Eldridge, G. R., and Hultgren, S. J. (2008). The biology and future prospects of antivirulence therapies. Nat. Rev. Microbiol. 6, 17-27. doi: $10.1038 /$ nrmicro2244

Clatworthy, A. E., Pierson, E., and Hung, D. T. (2007). Targeting virulence: a new paradigm for antimicrobial therapy. Nat. Chem. Biol. 3, 541-548. doi 10.1038/nchembio.2007.24

Clement, A., Tamalet, A., Leroux, E., Ravilly, S., Fauroux, B., and Jais, J. P. (2006). Long term effects of azithromycin in patients with cystic fibrosis: a double blind, placebo controlled trial. Thorax 61, 895-902. doi: 10.1136/thx.2005. 057950

Coggan, K. A., and Wolfgang, M. C. (2012). Global regulatory pathways and cross-talk control Pseudomonas aeruginosa environmental lifestyle and virulence phenotype. Curr. Issues Mol. Biol. 14, 47-70.

Driscoll, J. A., Brody, S. L., and Kollef, M. H. (2007). The epidemiology, pathogenesis and treatment of Pseudomonas aeruginosa infections. Drugs 67, 351-368. doi 10.2165/00003495-200767030-00003

Equi, A., Balfour-Lynn, I. M., Bush, A., and Rosenthal, M. (2002). Long term azithromycin in children with cystic fibrosis: a randomised, placebocontrolled crossover trial. Lancet 360, 978-984. doi: 10.1016/S0140-6736(02) 11081-6

Favre-Bonté, S., Köhler, T., and Van Delden, C. (2003). Biofilm formation by Pseudomonas aeruginosa: role of the C4-HSL cell-to-cell signal and inhibition by azithromycin. J. Antimicrob. Chemother. 52, 598-604. doi: 10.1093/jac/ dkg397

Fernebro, J. (2011). Fighting bacterial infections - future treatment options. Drug Resist. Updat. 14, 125-139. doi: 10.1016/j.drup.2011.02.001

Ferrer, I. R., Araki, K., and Ford, M. L. (2011). Paradoxical aspects of rapamycin immunobiology transplantation. Am. J. Transplant. 11, 654-659. doi: 10.1111/j.1600-6143.2011.03473

Fleet, J. E., Guha, K., Piper, S., Banya, W., Bilton, D., and Hodson, M. E. (2013). A retrospective analysis of the impact of azithromycin maintenance therapy on adults attending a UK cystic fibrosis clinic. J. Cyst. Fibros. 12, 49-53. doi: 10.1016/j.jcf.2012.05.010
Foulds, G., Shepard, R. M., and Johnson, R. B. (1990). The pharmacokinetics of azithromycin in human serum and tissues. J. Antimicrob. Chemother. 25(Suppl. A):73-82. doi: 10.1093/jac/25.suppl_A.73

Frangipani, E., Visaggio, D., Heeb, S., Kaever, V., Cámara, M., Visca, P., et al. (2014). The Gac/Rsm and cyclic-di-GMP signalling networks coordinately regulate iron uptake in Pseudomonas aeruginosa. Environ. Microbiol. 16, 676-688. doi: $10.1111 / 1462-2920.12164$

Giamarellos-Bourboulis, E. J. (2008). Immunomodulatory therapies for sepsis: unexpected effects with macrolides. Int. J. Antimicrob. Agents 32(Suppl.1):S39S43. doi: 10.1016/j.ijantimicag.2008.06.004

Gillis, R. J., and Iglewski, B. H. (2004). Azithromycin retards Pseudomonas aeruginosa biofilm formation. J. Clin. Microbiol. 42, 5842-5845. doi: 10.1128/JCM.42.12.5842-5845.2004

Girard, A. E., Girard, D., English, A. R., Gootz, T. D., Cimochowski, C. R., Faiella, J. A., etal. (1987). Pharmacokinetic and in vivo studies with azithromycin (CP-62,993), a new macrolide with an extended half-life and excellent tissue distribution. Antimicrob. Agents Chemother. 31, 1948-1954. doi: 10.1128/AAC.31.12.1948

Gödeke, J., Pustelny, C., and Häussler, S. (2013). Recycling of peptidyl-tRNAs by peptidyl-tRNA hydrolase counteracts azithromycin-mediated effects on Pseudomonas aeruginosa. Antimicrob. Agents Chemother. 57, 1617-1624. doi: 10.1128/AAC.02582-12

Hicks, L. A., Taylor, T. H., and Hunkler, R. J. (2013). U.S. outpatient antibiotic prescribing, 2010. N. Engl. J. Med. 368, 1461-1462. doi: 10.1056/NEJMc1212055

Hodge, S., Hodge, G., Jersmann, H., Matthews, G., Ahern, J., Holmes, M., et al. (2008). Azithromycin improves macrophage phagocytic function and expression of mannose receptor in chronic obstructive pulmonary disease. Am. J. Respir. Crit. Care Med. 178, 139-148. doi: 10.1164/rccm.200711$1666 \mathrm{OC}$

Hoffmann, N., Lee, B., Hentzer, M., Rasmussen, T. B., Song, Z., Johansen, H. K., et al. (2007). Azithromycin blocks quorum sensing and alginate polymer formation and increases the sensitivity to serum and stationary-growth-phase killing of Pseudomonas aeruginosa and attenuates chronic P. aeruginosa lung infection in Cftr(-/-). mice. Antimicrob. Agents Chemother. 51, 3677-3687. doi: 10.1128/AAC.01011-06

Ichimiya, T., Takeoka, K., Hiramatsu, K., Hirai, K., Yamasaki, T., and Nasu, M. (1996). The influence of azithromycin on the biofilm formation of Pseudomonas aeruginosa in vitro. Chemotherapy 42, 186-191. doi: 10.1159/0002 39440

Imamura, Y., Higashiyama, Y., Tomono, K., Izumikawa, K., Yanagihara, K., Ohno, H., etal. (2005). Azithromycin exhibits bactericidal effects on Pseudomonas aeruginosa through interaction with the outer membrane. Antimicrob. Agents Chemother. 49, 1377-1380. doi: 10.1128/AAC.49.4.1377-1380. 2005

Imperi, F., Massai, F., Facchini, M., Frangipani, E., Visaggio, D., Leoni, L., et al. (2013). Repurposing the antimycotic drug flucytosine for suppression of Pseudomonas aeruginosa pathogenicity. Proc. Natl. Acad. Sci. U.S.A. 110, 7458-7463. doi: 10.1073/pnas.1222706110

Itkin, I. H., and Menzel, M. L. (1970). The use of macrolide antibiotic substances in the treatment of asthma. J. Allergy 45, 146-162. doi: 10.1016/00218707(70)90124-3

Jaffé, A., Francis, J., Rosenthal, M., and Bush, A. (1998). Long-term azithromycin may improve lung function in children with cystic fibrosis. Lancet 351, 420. doi: 10.1016/S0140-6736(05)78360-4

Kai, T., Tateda, K., Kimura, S., Ishii, Y., Ito, H., Yoshida, H., et al. (2009). A low concentration of azithromycin inhibits the mRNA expression of $\mathrm{N}$-acyl homoserine lactone synthesis enzymes, upstream of lasI or rhlI, in Pseudomonas aeruginosa. Pulm. Pharmacol. Ther. 22, 483-486. doi: 10.1016/j.pupt.2009. 04.004

Kanoh, S., and Rubin, B. K. (2010). Mechanisms of action and clinical application of macrolides as immunomodulatory medications. Clin. Microbiol. Rev. 23, 590615. doi: 10.1128/CMR.00078-09

Kita, E., Sawaki, M., Oku, D., Hamuro, A., Mikasa, K., Konishi, M., et al. (1991). Suppression of virulence factors of Pseudomonas aeruginosa by erythromycin. J. Antimicrob. Chemother. 27, 273-284. doi: 10.1093/jac/27.3.273

Kohler, T., Dumas, J. L., and Van Delden, C. (2007). Ribosome protection prevents azithromycin-mediated quorum-sensing modulation and stationary phase killing of Pseudomonas aeruginosa. Antimicrob. Agents Chemother. 51, 4243-4248. doi: 10.1128/AAC.00613-07 
Law, G. L., Tisoncik-Go, J., Korth, M. J., and Katze, M. G. (2013). Drug repurposing: a better approach for infectious disease drug discovery? Curr. Opin. Immunol. 25 , 588-592. doi: 10.1016/j.coi.2013.08.004

Legssyer, R., Huaux, F., Lebacq, J., Delos, M., Marbaix, E., Lebecque, P., et al. (2006). Azithromycin reduces spontaneous and induced inflammation in DeltaF508 cystic fibrosis mice. Respir. Res. 7, 134. doi: 10.1186/1465-99217-134

Lutz, L., Pereira, D. C., Paiva, R. M., Zavascki, A. P., and Barth, A. L. (2012) Macrolides decrease the minimal inhibitory concentration of anti-pseudomonal agents against Pseudomonas aeruginosa from cystic fibrosis patients in biofilm. BMC Microbiol. 12:196. doi: 10.1186/1471-2180-12-196

McAlister, V. C., Mahalati, K., Peltekian, K. M., Fraser, A., and MacDonald, A. S. (2002). A clinical pharmacokinetic study of tacrolimus and sirolimus combination immunosuppression comparing simultaneous to separated administration. Ther. Drug Monit. 24, 346-350. doi: 10.1097/00007691-20020600000004

Miyairi, S., Tateda, K., Fuse, E. T., Ueda, C., Saito, H., Takabatake, T., et al. (2006). Immunization with 3-oxododecanoyl-L-homoserine lactone-protein conjugate protects mice from lethal Pseudomonas aeruginosa lung infection. J. Med. Microbiol. 55, 1381-1387. doi: 10.1099/jmm.0.46658-0

Molinari, G., Guzman, C. A., Pesce, A., and Schito, G. C. (1993). Inhibition of Pseudomonas aeruginosa virulence factors by subinhibitory concentrations of azithromycin and other macrolide antibiotics. J. Antimicrob. Chemother. 31, 681-688. doi: 10.1093/jac/31.5.681

Molinari, G., Paglia, P., and Schito, G. C. (1992). Inhibition of motility of Pseudomonas aeruginosa and proteus mirabilis by subinhibitory concentrations of azithromycin. Eur. J. Clin. Microbiol. Infect. Dis. 11, 469-471. doi: $10.1007 / \mathrm{BF} 01961867$

Morita, Y., Kimura, N., Mima, T., Mizushima, T., and Tsuchiya, T. (2001). Roles of MexXY-and MexAB multidrug efflux pumps in intrinsic multidrug resistance of Pseudomonas aeruginosa PAO1. J. Gen. Appl. Microbiol. 47, 27-32. doi: 10.2323 /jgam. 47.27

Nagino, K., and Kobayashi, H. (1997). Influence of macrolides on mucoid alginate biosynthetic enzyme from Pseudomonas aeruginosa. Clin. Microbiol. Infect. 3 , 432-439. doi: 10.1111/j.1469-0691.1997.tb00279.x

Nalca, Y., Jänsch, L., Bredenbruch, F., Geffers, R., Buer, J., and Häussler, S. (2006). Quorum-sensing antagonistic activities of azithromycin in Pseudomonas aeruginosa PAO1: a global approach. Antimicrob. Agents Chemother. 50, 1680-1688. doi: 10.1128/AAC.50.5.1680-1688.2006

Nguyen, D., Emond, M. J., Mayer-Hamblett, N., Saiman, L., Marshall, B. C., and Burns, J. L. (2007). Clinical response to azithromycin in cystic fibrosis correlates with in vitro effects on Pseudomonas aeruginosa phenotypes. Pediatr. Pulmonol. 42, 533-541. doi: 10.1002/ppul.20620

Nicolau, D. P., Banevicius, M. A., Marangos, M. N., Klepser, M. E., Quintiliani, R., and Nightingale, C. H. (1997). Influence of adjunct azithromycin on the mortality of experimental Pseudomonas aeruginosa sepsis. Int. J. Antimicrob. Agents 8, 239241. doi: 10.1016/S0924-8579(97)00017-4

Nicolau, D. P., Banevicius, M. A., Nightingale, C. H., and Quintiliani, R. (1999). Beneficial effect of adjunctive azithromycin in treatment of mucoid Pseudomonas aeruginosa pneumonia in the murine model. Antimicrob. Agents Chemother. 43, 3033-3035.

Pal, S. (2006). A journey across the sequential development of macrolides and ketolides related to erythromycin. Tetrahedron 62, 3171-3200. doi: 10.1016/j.tet.2005.11.064

Pérez-Martínez, I., and Haas, D. (2011). Azithromycin inhibits expression of the GacA-dependent small RNAs RsmY and RsmZ in Pseudomonas aeruginosa. Antimicrob. Agents Chemother. 55, 3399-3405. doi: 10.1128/AAC.018 01-10

Poehlsgaard, J., and Douthwaite, S. (2005). The bacterial ribosome as a target for antibiotics. Nat. Rev. Microbiol. 3, 870-881. doi: 10.1038/nrmicr 01265

Rasko, D. A., and Sperandio, V. (2010). Anti-virulence strategies to combat bacteria-mediated disease. Nat. Rev. Drug Discov. 9, 117-128. doi: 10.1038/nr d 3013

Ratjen, F., Saiman, L., Mayer-Hamblett, N., Lands, L. C., Kloster, M., Thompson, V., et al. (2012). Effect of azithromycin on systemic markers of inflammation in patients with cystic fibrosis uninfected with Pseudomonas aeruginosa. Chest 142, 1259-1266. doi: 10.1378/chest.12-0628
Retsema, J., and Fu, W. (2001). Macrolides: structures and microbial targets. Int. J. Antimicrob. Agents 18(Suppl. 1):S3-S10. doi: 10.1016/S0924-8579(01)00401-0

Retsema, J., Girard, A., Schelkly, W., Manousos, M., Anderson, M., Bright, G., et al. (1987). Spectrum and mode of action of azithromycin (CP-62,993), a new 15-membered-ring macrolide with improved potency against gramnegative organisms. Antimicrob. Agents Chemother. 31, 1939-1947. doi: 10.1128/AAC.31.12.1939

Saiman, L., Anstead, M., Mayer-Hamblett, N., Lands, L. C., Kloster, M., HocevarTrnka, J., et al. (2010). Effect of azithromycin on pulmonary function in patients with cystic fibrosis uninfected with Pseudomonas aeruginosa: a randomized controlled trial. JAMA 303, 1707-1715. doi: 10.1001/jama.2010.563

Saiman, L., Marshall, B. C., Mayer-Hamblett, N., Burns, J. L., Quittner, A. L., Cibene, D. A., et al. (2003). Azithromycin in patients with cystic fibrosis chronically infected with Pseudomonas aeruginosa: a randomized controlled trial. JAMA 290, 1749-1756. doi: 10.1001/jama.290.13.1749

Salmond, R. J., and Zamoyska, R. (2011). The influence of mTOR on T helper cell differentiation and dendritic cell function. Eur. J. Immunol. 41, 2137-2141. doi: 10.1002/eji.201141523

Schultz, M. J. (2004). Macrolide activities beyond their antimicrobial effects: macrolides in diffuse panbronchiolitis and cystic fibrosis. J. Antimicrob. Chemother. 54, 21-28. doi: 10.1093/jac/dkh309

Skindersoe, M. E., Alhede, M., Phipps, R., Yang, L., Jensen, P. O., Rasmussen, T. B., et al. (2008). Effects of antibiotics on quorum sensing in Pseudomonas aeruginosa. Antimicrob. Agents Chemother. 52, 3648-3663. doi: 10.1128/AAC.01230-07

Southern, K. W., Barker, P. M., Solis-Moya, A., and Patel, L. (2012). Macrolide antibiotics for cystic fibrosis. Cochrane Database Syst. Rev. 2004, CD002203. doi: 10.1002/14651858.CD002203.pub4

Spreer, A., Kerstan, H., Böttcher, T., Gerber, J., Siemer, A., Zysk, G., et al. (2003). Reduced release of pneumolysin by Streptococcus pneumoniae in vitro and in vivo after treatment with nonbacteriolytic antibiotics in comparison to ceftriaxone. Antimicrob. Agents Chemother. 47, 2649-2654. doi: 10.1128/AAC.47.8.26492654.2003

Steel, H. C., Theron, A. J., Cockeran, R., Anderson, R., and Feldman, C. (2012). Pathogen- and host-directed anti-inflammatory activities of macrolide antibiotics. Mediators Inflamm. 2012, 5842-5862. doi: 10.1155/2012/584262

Sugimura, M., Maseda, H., Hanaki, H., and Nakae, T. (2008). Macrolide antibiotic-mediated downregulation of MexAB-OprM efflux pump expression in Pseudomonas aeruginosa. Antimicrob. Agents Chemother. 52, 4141-4144. doi: 10.1128/AAC.00511-08

Tateda, K., Comte, R., Pechere, J. C., Kohler, T., Yamaguchi, K., and Van Delden, C. (2001). Azithromycin inhibits quorum sensing in Pseudomonas aeruginosa. Antimicrob. Agents Chemother. 45, 1930-1933. doi: 10.1128/AAC.45.6.19301933.2001

Tateda, K., Hirakata, Y., Furuya, N., Ohno, A., and Yamaguchi, K. (1993). Effects of sub-MICs of erythromycin and other macrolide antibiotics on serum sensitivity of Pseudomonas aeruginosa. Antimicrob. Agents Chemother. 37, 675-680. doi: 10.1128/AAC.37.4.675

Tateda, K., Ishii, Y., Hirakata, Y., Matsumoto, T., Ohno, A., and Yamaguchi, K. (1994). Profiles of outer membrane proteins and lipopolysaccharide of Pseudomonas aeruginosa grown in the presence of sub-MICs of macrolide antibiotics and their relation to enhanced serum sensitivity. J. Antimicrob. Chemother. 34, 931-942. doi: 10.1093/jac/34.6.931

Tateda, K., Ishii, Y., Matsumoto, T., Furuya, N., Nagashima, M., Matsunaga, T., et al. (1996). Direct evidence for antipseudomonal activity of macrolides: exposure-dependent bactericidal activity and inhibition of protein synthesis by erythromycin, clarithromycin, and azithromycin. Antimicrob. Agents Chemother. 40, 2271-2275.

Tsai, W. C., Hershenson, M. B., Zhou, Y., and Sajjan, U. (2009). Azithromycin increases survival and reduces lung inflammation in cystic fibrosis mice. Inflamm. Res. 58, 491-501. doi: 10.1007/s00011-009-0015-9

van Delden, C., Köhler, T., Brunner-Ferber, F., François, B., Carlet, J., and Pechère, J. C. (2012). Azithromycin to prevent Pseudomonas aeruginosa ventilatorassociated pneumonia by inhibition of quorum sensing: a randomized controlled trial. Intensive Care Med. 38, 1118-1125. doi: 10.1007/s00134-012-2559-3

Wargo, M. J., Gross, M. J., Rajamani, S., Allard, J. L., Lundblad, L. K., Allen, G. B., et al. (2011). Hemolytic phospholipase $\mathrm{C}$ inhibition protects lung function during Pseudomonas aeruginosa infection. Am. J. Respir. Crit. Care Med. 184, 345-354. doi: $10.1164 / \mathrm{rccm} .201103-0374 \mathrm{OC}$ 
Washington, J. A., and Wilson, W. R. (1985). Erythromycin: a microbial and clinical perspective after 30 years of clinical use (2). Mayo Clin. Proc. 60, 271-278. doi: 10.1016/S0025-6196(12)60219-5

Wei, Q., and Ma, L. Z. (2013). Biofilm matrix and its regulation in Pseudomonas aeruginosa. Int. J. Mol. Sci. 14, 20983-21005. doi: 10.3390/ijms141020983

Wilms, E. B., Touw, D. J., and Heijerman, H. G. (2006). Pharmacokinetics of azithromycin in plasma, blood, polymorphonuclear neutrophils and sputum during long-term therapy in patients with cystic fibrosis. Ther. Drug Monit. 28, 219-225. doi: 10.1097/01.ftd.0000195617.69721.a5

Conflict of Interest Statement: The authors declare that the research was conducted in the absence of any commercial or financial relationships that could be construed as a potential conflict of interest.
Received: 18 February 2014; accepted: 31 March 2014; published online: 22 April 2014. Citation: Imperi F, Leoni L and Visca P (2014) Antivirulence activity of azithromycin in Pseudomonas aeruginosa. Front. Microbiol. 5:178. doi: 10.3389/fmicb.2014. 00178

This article was submitted to Antimicrobials, Resistance and Chemotherapy, a section of the journal Frontiers in Microbiology.

Copyright (c) 2014 Imperi, Leoni and Visca. This is an open-access article distributed under the terms of the Creative Commons Attribution License (CC BY). The use, distribution or reproduction in other forums is permitted, provided the original author(s) or licensor are credited and that the original publication in this journal is cited, in accordance with accepted academic practice. No use, distribution or reproduction is permitted which does not comply with these terms. 